\title{
Avaliação in vitro da estabilidade de cor de resinas compostas bulk-fill
}

An in vitro evaluation of the color stability of bulk-fill resin composites

Evaluación in vitro de la estabilidad del color de resinas compuestas bulk-fill

Anne Gabrielle dos Santos Mesquita MOURA ${ }^{1}$

Maria Gabriella Rodrigues Rafael de MELO ${ }^{1}$

Wanderson Talles do Nascimento Pereira SANTOS ${ }^{2}$

Ana Luisa Cassiano Alves BEZERRA ${ }^{3}$

Gabriela Queiroz de Melo MONTEIRO ${ }^{4}$

Márcia de Almeida DURÃO 5

${ }^{1}$ Graduado(a) em Odontologia, Centro Universitário Mauricio de Nassau - UNINASSAU/ PE, 52011-000 Recife-PE, Brasil

${ }^{2}$ Programa de Pós-Graduação em Ciências Odontológicas, Centro de Ciências da Saúde, Universidade Federal do Rio Grande do Norte - UFRN 59012-570 Natal-RN, Brasil

${ }^{3}$ Mestranda em Clínica Odontológica, Faculdade de Odontologia, Universidade de Pernambuco- FOP/UPE, 50100-010 Camaragibe-PE, Brasil ${ }^{4}$ Doutora em Dentística, Professora Associada da Faculdade de Odontologia, Universidade de Pernambuco - FOP/UPE, 50100-010 Camaragibe/PE, Brasil

${ }^{5}$ Pós-Doutoranda em Dentística, Faculdade de Odontologia, Universidade de Pernambuco- FOP/UPE, 50.100-010, Camaragibe-PE, Brasil Docente do Curso de Odontologia, Centro Universitário Mauricio de Nassau - UNINASSAU/ PE, 52011-000, Recife-PE, Brasil

\section{Resumo}

Introdução: A odontologia restauradora vem evoluindo em busca de materiais restauradores que mimetizem a estrutura dentária e seus efeitos ópticos, sendo um grande desafio nas pesquisas dos materiais dentários. Objetivo: Avaliar e comparar a alteração cromática de três resinas bulk-fill (Tetric EvoCeram Bulk Fill; Aura Bulk Fill; Filtek Bulk Fill) e uma resina convencional (Filtek Z250) após a exposição diária ao café num período de 28 dias. Materiais e métodos: 80 corpos de prova foram confeccionados, divididos em 4 grupos de cada resina estudada $(n=20)$, armazenados em água destilada, trocada diariamente. E em 8 subgrupos $(n=10)$, sendo 4 experimentais (de cada resina), submetidos à imersão diária de 20 minutos em solução de café durante 28 dias e 4 subgrupos controle mantidos em água destilada. A tomada de cor foi realizada no baseline, 7,14 e 28 dias, por meio do método subjetivo (Escala Vita Classical), por 2 avaliadores em consenso e também no baseline e após 28 dias pelo método objetivo (Vita Easy Shade). Resultados: Análise comparativa entre os grupos controle e experimentais demonstrou alteração de cor em todas as resinas compostas analisadas. O café promoveu manchamento significativo em todos os tempos, nas avaliações subjetivas e objetivas. Conclusão: As resinas compostas bulk-fill apresentaram estabilidade de cor de semelhante a superior à resina composta convencional frente à exposição diária ao café, ressaltando-se a importância da realização de acabamento e polimento para minimizar manchamento.

Descritores: Materiais Dentários; Resinas Compostas; Estética Dentária; Dentística Operatória.

\section{Abstract}

Introduction: Restorative dentistry has been evolving in seach of restorative materials that mimic the dental structure and its optical effects, being a great challenge in the research of dental materials. Objective: To evaluate and compare the chromatic alteration of three bulk-fill resins (Tetric EvoCeram Bulk Fill; Filtek Bulk Fill) and a conventional resin (Filtek Z250) after daily exposure to coffee in a period of 28 days. Materials and methods: 80 specimens were prepared, divided into 4 groups of each resin studied $(n=20)$, stored in distilled water, changed daily, and these divided into 8 subgroups $(n=10)$, 4 experimental (from each resin) were submitted to daily immersion of 20 minutes in coffee solution for 28 days and 4 control subgroups maintained in distilled water. The color selection was performed at baseline, 7, 14 and 28 days, using the subjective method (Vita Classical Scale), by two evaluators in consensus and also at baseline and after 28 days by the objective method (Vita Easy Shade). Results: Comparative analysis between control and experimental groups showed color change in all resin composites analyzed. Coffee promoted significant staining at all times, in subjective and objective assessments. Conclusion: The bulk-fill composites showed color stability similar to superior of conventional composite resin compared to daily coffee exposure, emphasizing the importance of finishing and polishing to minimize staining.

Descriptors: Dental Materials; Composite Resins; Esthetic Dental; Dentistry Operative.

\section{Resumen}

Introducción: La odontologia restauradora ha evolucionado en busca de materiales restauradores que imiten la estrutura dental y sus efectos ópticos, siendo un gran desafio en la investigación de materiales dentales. Objetivo: evaluar y comparar la alteración cromática de tres resinas Bulk-Fill (Tetric EvoCeram Bulk Fill; Aura Bulk Fill; Filtek Bulk Fill) y uma resina convencional (Filtek Z250) después de la exposición diária al café durante um período de 28 días. Materiales y métodos: se hicieron 80 muestras divididas em 4 grupos de cada resina estudiada $(n=20)$, almacenadas em agua destilada , cambiadas diariamente. $Y$ em 8 subgrupos $(n=10), 4$ de los cuales fueron experimentales (de casa resina), sometidos a uma inmersión diária de 20 minutos em solución de café durante 28 días y 4 subgrupos de control mantenidos em agua destilada. El color fue tomado al inicio del estúdio, 7, 14 y 28 días, utilizando el método subjetivo (Escala clássina de Vita), por 2 evaluadores de consenso y también al inicio del estúdio, y después de 28 días utilizando el método objetivo (Vita Easy Shade). Resultados: El análisis comparativo entre los grupos control y experimental mostro um cambio de color em todas las resinas compuestas analizadas. El café promovió um tinción significativa em todo momento, em evaluacionas subjetivas y objetivas. Conclusión: Las resinas compuestas de relleno a granel mostraton uma estabilidade del color similar a la de las resinas compuestas convencionales frente a la exposición diária al café, enfatizando la importância del acabado y pulido para minimizar las manchas.

Descriptores: Materiales Dentales; Resinas Compuestas; Estética Dental; Odontología Operativa.

\section{INTRODUÇÃo}

A Odontologia Restauradora vem evoluindo em busca do material restaurador ideal, que mimetizem a estrutura dentária e seus efeitos ópticos, sendo um grande desafio nas pesquisas dos materiais dentários ${ }^{1}$.

Em 1962, Dr. Ray L. Bowen desenvolveu o monômero bisfenol A-glicidil Metacrilato (Bis$(\mathrm{GMA})^{2}$, e posteriormente uniu-o à resina composta ${ }^{3}$, tornando-a o material de escolha em restaurações estéticas a partir da década de 70 até os dias atuais. Entretanto, sabe-se que as resinas compostas tendem a sofrer 
descoloração devido a retenção de pigmentos por parte da sua superfície rugosa, portanto, um acabamento e polimento criterioso são fundamentais para a redução da rugosidade superficial e consequentemente do manchamento superficial das restaurações ${ }^{4,5}$.

A escolha do tipo de resina pode influenciar de forma considerável o resultado da cor da restauração em longo prazo. Sabe-se que o tamanho das partículas de carga pode influenciar na lisura da superfície da restauração, as de partículas pequenas proporcionam uma de lisura razoável, não superando a lisura obtida nas resinas microparticuladas ou das híbridas, sendo estas últimas muito usadas pela boa combinação de suas características físicas e mecânicas ${ }^{1}$.

A estabilidade da cor tem se tornado fundamental para a escolha do material restaurador, estando a descoloração entre os principais motivos para substituição da restauração ${ }^{6-8}$. Estudos comprovaram a influência de alguns alimentos na alteração de cor sofrida por restaurações em resina composta ${ }^{4,9,10}$, pois o manchamento extrínseco também pode ser influenciado pela capacidade de absorção de líquidos apresentadas pelo material restaurador ${ }^{11,12}$.

As resinas compostas convencionais exigem técnica de inserção incremental, respeitando-se o Fator $\mathrm{C}$ (Fator de configuração cavitária), devido ao fato que durante sua fotopolimerização, ocorre uma contração dos monômeros, o que pode gerar tensões de contração que possibilitam o surgimento de microtrincas/microfraturas, fendas, perda de integridade marginal, microinfiltrações, sensibilidade pós-operatória, desgaste superficial e cárie secundária ${ }^{13-17}$. Essa técnica incremental, utilizada nas resinas composta convencionais aumenta o torna o tempo clínico (paciente e odontólogo), há maior chances de falhas por parte do operador, por ser uma técnica mais complexa, que exige muito conhecimento e habilidade.

Em 2010, foi lançada no mercado uma nova geração de resinas compostas chamadas Bulk-Fill, com a proposta de inserção em incremento único de até 4 ou $5 \mathrm{~mm}$ (de acordo com fabricante). Inicialmente, surgiram as do tipo flow, com indicação para preenchimento da base de cavidades muito profundas, e posteriormente foram desenvolvidas em consistência regular, passível de escultura dental ${ }^{18}$. Essa técnica de incremento único somente tornou-se possível devido a translucidez que essas resinas possuem, permitindo uma maior penetração de luz, e também pela incorporação de monômero especiais que reduzem 0 stress de polimerização ${ }^{19}$.

O objetivo deste estudo foi avaliar e comparar a estabilidade de cor, em diferentes períodos de tempo, de uma resina composta convencional e três resinas do tipo Bulk-Fill, mediante exposição à substância corante (café). A hipótese nula testada foi de que não haveria diferença entre os grupos estudados.

MATERIAL E MÉTODO

Foram confeccionados o total de 80 corpos de prova cilíndricos com o auxílio de uma matriz de silicone, com $4 \mathrm{~mm}$ de diâmetro e $2 \mathrm{~mm}$ de espessura. As resinas compostas testadas, Tetric EvoCeram Bulk Fill (Ivoclar Vivadent, Amherst, NY, USA); Filtek Bulk Fill (3M ESPE, St. Paul, MN, USA), Aura Bulk Fill (SDI, Bayswater, Victoria, Australia) e Filtek Z250 (3M ESPE, St. Paul, MN, USA), foram inseridas na matriz segundo instruções de seus fabricantes. Após preenchimento da matriz, foi colocado sobre a resina uma tira de poliéster e uma lamínula de vidro, e exercida uma pressão digital durante 20 segundos para garantir melhor acomodação do material $\left.\right|^{5,20-23}$.

A fotoativação foi realizada com aparelho LED Polywave, Bluephase (Ivoclar Vivadent, Amherst, Nova York, USA), com condutor de 10 $\mathrm{mm}$ de diâmetro. Foi utilizado na opção High Power Program (cerca de $1.500 \mathrm{~mW} / \mathrm{cm} 2$ ) pelo tempo indicado pelo fabricante e sua densidade de potência foi aferida periodicamente durante $o$ estudo como radiômetro. Os excessos das bordas dos corpos de prova foram removidos com lâmina de bisturi $\mathrm{n}^{\circ} 12$ e regularizados com lixas d'água (\#600 e \#1200). Após 24h em água destilada, as faces superiores de todos os corpos de prova foram polidas na sequência com Pontas Astropol (Ivoclar Vivadent) de média e baixa granulação e finalizado com Escova de Carbeto de Silício Astrobrush (Ivoclar Vivadent), em baixa rotação, com movimentos intermitentes em sentido único por 20 segundos. Assim a superfície foi regularizada e polida, conferindo lisura e brilho à camada superficial, reduzindo possibilidades de degradação precoce, alteração de cor e rugosidade. A espessura e diâmetro de cada corpo de prova foram aferidos em quatro pontos utilizando um paquímetro digital (Digimess, São Paulo, SP, Brasil), lavados e em seguida foram secos em papel absorvente.

Foram divididos em 8 grupos $(n=10)$, sendo 4 grupos, $F$ (Filtek Bulk Fill), T (Tetric EvoCeram Bulk Fill), A (Aura Bulk Fill), Z (Z250) mantidos apenas em água destilada, trocada 
diariamente e os 4 grupos experimentais Fc, Tc, Ac e Zc submetidos a imersões diárias de 20 minutos em solução de café na proporção de $250 \mathrm{ml}$ de água fervente para $25 \mathrm{~g}$ de Café solúvel (Nescafé Tradição, Nestlé, Araras, São Paulo, Brasil) ${ }^{24}$, sendo estes também mantidos em água destilada no intervalo entre os ciclos.

A tomada de cor foi realizada no baseline, 7, 14 e 28 dias através de consenso, por 2 (dois) avaliadores calibrados, para 0 método subjetivo (Escala Vita Classical), e no baseline e após 28 dias por meio do método objetivo com espectrofotômetro clínico (Vita EasyShade ${ }^{\circledR}$ - Vident - Brea, CA, EUA).

RESULTADOS

Os resultados obtidos na avaliação cromática dos 80 corpos de prova nos diferentes tempos estudados, por meio dos métodos subjetivo e objetivo, no topo dos corpos de prova onde foi realizado acabamento e polimento e na base sem acabamento e polimento, estão Tabela 1 .

Tabela 1. Resultados de avaliação cromática das resinas compostas, nos diferentes métodos e tempos

\begin{tabular}{|c|c|c|c|c|c|c|c|}
\hline \multicolumn{8}{|c|}{ RESINA/TEMPO/MÉTODO/COR } \\
\hline \multirow[t]{3}{*}{ Compósito/Imersão } & \multicolumn{2}{|c|}{ Baseline } & \multirow{3}{*}{$\begin{array}{c}7 \\
\text { Dias } \\
\text { vITA }\end{array}$} & \multirow{3}{*}{$\begin{array}{c}14 \\
\text { Dias } \\
\text { VITA } \\
\end{array}$} & \multicolumn{3}{|c|}{28 Dias } \\
\hline & \multirow{2}{*}{$\begin{array}{l}\text { EASY } \\
\text { SHADE }\end{array}$} & \multirow[b]{2}{*}{ VITA } & & & \multirow{2}{*}{$\begin{array}{l}\text { EASY } \\
\text { SHADE }\end{array}$} & \multicolumn{2}{|c|}{ VITA } \\
\hline & & & & & & TOPO & BASE \\
\hline $\begin{array}{l}\text { TETRIC EVOCERAM } \\
\text { BULK FILL / CAFÉ }\end{array}$ & B1 & A1 & $\mathrm{C}_{3}$ & $\mathrm{~A}_{3}$ & A3 & $\mathrm{C} 2$ & $\mathrm{C}_{4}$ \\
\hline $\begin{array}{c}\text { TETRIC EVOCERAM } \\
\text { BULK FILL / ÁGUA }\end{array}$ & B1 & A1 & $\mathrm{C} 1$ & B1 & A1 & B1 & B1 \\
\hline AURA BULK FILL / & A1 & A1 & $\mathrm{C}_{4}$ & B2 & A3 & $\mathrm{C}_{2}$ & A4 \\
\hline $\begin{array}{c}\text { AURA BULK FILL / } \\
\text { ÁGUA }\end{array}$ & A1 & A1 & $\mathrm{C} 1$ & B1 & A2 & A & A1 \\
\hline $\begin{array}{c}\text { FILTEK BULK FILL / } \\
\text { CAFÉ }\end{array}$ & A1 & A1 & $\mathrm{C}_{4}$ & $\mathrm{C}_{2}$ & A3,5 & $\mathrm{C}_{3}$ & $\mathrm{C}_{4}$ \\
\hline $\begin{array}{c}\text { FILTEK BULK FILL / } \\
\text { ÂGUA }\end{array}$ & A1 & A1 & $\mathrm{C} 1$ & A1 & B1 & B1 & B1 \\
\hline FILTEK Z 250 / CAFÉ & A1 & A1 & A3,5 & A3,5 & $\mathrm{A} 3,5$ & $\mathrm{C}_{3}$ & $\mathrm{C}_{4}$ \\
\hline FILTEK Z 250 / ÁGUA & A1 & A1 & $\mathrm{C} 1$ & A2 & $\mathrm{A}_{3}$ & A2 & A2 \\
\hline
\end{tabular}

Não houve diferença na análise da cor dos corpos de prova dos mesmos grupos $(n=10)$ nos diferentes tempos, em cada método. Foi realizada uma análise comparativa entre as amostras do grupo controle (mantidos em água destilada) e experimental (submetidos à imersão em café) de cada resina observando-se que ocorreu alteração de cor entre os grupos em todas as resinas analisadas, onde 0 café promoveu manchamento significativo em todos os tempos, nas avaliações subjetivas e objetivas.

A resina composta convencional, Filtek Z250 (3M ESPE) apresentou expressiva alteração, quando comparada com seu grupo controle. E quando comparada às resinas bulkfill, houve pigmentação semelhante à da resina Filtek Bulk-fill (3M ESPE), através de ambos os métodos, na análise de 28 dias.

Dentre as Resinas Bulk-Fill submetidas a solução de café, de acordo com o método subjetivo, os grupos da resina composta Aura Bulk Fill e da Tetric EvoCeram Bulk Fill apresentaram o menor grau de alteração de cor (C2) em detrimento ao grupo da Filtek Bulk Fill (C3), apesar desta diferença de cor ser discreta. De acordo com o método objetivo, embora tenha ocorrido diferença das cores encontradas entre os métodos, os grupos Tetric EvoCeram Bulk Fill e Aura Bulk Fill permaneceram com menor alteração de coloração (A3), enquanto que o grupo Filtek Bulk Fill $(A 3,5)$, continuou apresentando a maior alteração cromática.

Após 28 dias foram comparadas através do método subjetivo, as faces dos corpos de prova que foram submetidas ao processo de acabamento e polimento (TOPO), com as faces onde não foram realizados 0 acabamento e polimento (BASE), onde o topo dos corpos de prova apresentou menor manchamento.

DISCUSSÃO

A hipótese nula testada não foi rejeitada, uma vez que não houve diferença na cor dos corpos de prova dos mesmos grupos $(n=10)$ nos diferentes tempos, em cada método. Desse modo, pode-se admitir que a alteração cromática extrínseca dos materias resinosos restauradores está diretamente relacionada à dieta, aos hábitos de higiene bucal $e$ às propriedades químicas das resinas utilizadas, como teor da matriz orgânica, tamanho e quantidade das partículas de carga inorgânica e também 0 emprego do acabamento $e$ polimento $^{25,26}$.

A escolha da solução de café para este experimento se deu pelo fato deste ser considerado, em diversos estudos, como a solução com maior potencial de manchamento ${ }^{27-30}$ e por ser uma solução ingerida diariamente no Brasil e diversos países.

Lopes et al. ${ }^{31}$ verificaram em seu estudo que o café apresentou maior potencial de pigmentação quando comparado a outras soluções corantes. Sendo que associado a outras pesquisas que também verificaram essa capacidade de manchamento, o café mais uma vez ficou à frente das soluções corantes, quando comparado ao chá que também foi material de estudo frente aos itens incluídos no teste ${ }^{31}$

Para Salgado et al. ${ }^{32}$ a descoloração dos compósitos a base de resina, são provenientes do meio extrínseco e intrínseco, sendo esse último causado pelas alterações químicas no interior do material, como a lixiviação de monômeros não reagidos por reação de hidrólise. O café, sendo um dos responsáveis pela pigmentação desses compósitos, quando 
absorvido pela camada superficial destes, causa um manchamento do tipo extrínseco, onde não é possível a remoção desses pigmentos escuros pelos procedimentos de polimento. Deste modo, vale uma ressalva quanto a importância desse polimento de forma adequada e no tempo correto, o que vem relacionar ao nosso estudo os resultados obtidos nos corpos de prova, onde houve o polimento, 0 manchamento foi quase insignificante ${ }^{32}$.

Neste estudo foram utilizados para acabamento e polimento as pontas Astropol (Ivoclar Vivadent) na granulação média para remoção de excessos e fina para obtenção de superfícies mais lustrosas em especialmente em compósitos híbridos. Já o Astrobrush (Ivoclar Vivadent) é composto por carboneto de silício incorporado às cerdas, dispensando a pasta de polimento. Manhães ${ }^{33}$ observou que o sistema de polimento Astropol obteve superfícies mais lisas quando comparado a outros dois sistemas de acabamento e polimento, aplicados em resina microhíbrida.

As faces dos corpos de prova que foram submetidas ao processo de acabamento e polimento (TOPO) apresentaram menor manchamento, ressaltando a grande importância do acabamento e polimento. $O$ acúmulo de biofilme, o contato com alimentos ou substâncias corantes e a técnica de acabamento e polimento estão entre as principais causas da alteração da cor de restaurações em resina composta ${ }^{34}$. O emprego de adequado acabamento e polimento tem papel fundamental na redução do manchamento extrínseco do material restaurador, pois promove maior lisura através da remoção da camada mais superficial, rica em matriz orgânica ${ }^{35}$. Uma superfície áspera, sem acabamento e polimento, tende a descolorir com mais facilidade ${ }^{36}$ o que contrapõe os resultados obtidos pelo estudo de Oliveira et $\mathrm{al}_{.37}$ que não verificou diferenças significativas na alteração de cor de amostras polidas versus amostras não polidas em um estudo que analisou a estabilidade de cor de uma resina composta nanoparticulada após imersões diárias em sucos artificiais ${ }^{37}$.

Em relação a composição, quanto maior a porcentagem de carga inorgânica em volume e menor a partícula, mais resistentes a alteração cromática serão os compósitos ${ }^{38} \circ$ que reafirma os resultados obtidos neste estudo, tendo em vista que a Filtek Bulk Fill e a Z250 obtiveram as maiores alterações de cor ao passo que são as que apresentam menor \% por volume de carga inorgânica; e dentre os componentes orgânicos, o UDMA proporciona maior estabilidade de cor do que Bis-EMA, BisGMA e TEGDMA, devido ao seu potencial hidrofóbico $^{39}$. O que corrobora com o fato da resina composta Filtek Z250 ter apresentado o maior grau de manchamento, chamando atenção por ser a única com TEGDMA em sua composição, que é o componente orgânico mais hidrofílico ${ }^{40}$.

Outro fator que deve ser considerado é o tipo e a quantidade de fotoiniciador presentes na composição das resinas, bem como a qualidade da polimerização do compósito. O fotoiniciador mais utilizado atualmente é a canforoquinona e apesar de ser utilizada em baixas quantidades, há uma grande influência sob a cor do material por possuir uma cor amarelada que ao ser fotoativada muda para transparente ${ }^{41}$. A composição do material, o tipo de sistema fotoiniciador e a taxa de conversão do material desempenham um papel importante para a estabilidade das resinas impedindo que haja a degradação da matriz orgânica e a alteração no grau de absorção do material, evitando assim alterações de cor da restauração ${ }^{42,43}$.

CONCLUSÃO

De acordo com a metodologia proposta por este trabalho, concluiu-se que as resinas compostas Bulk Fill estudadas apresentaram estabilidade de cor semelhante a superior quando comparadas à resina composta convencional, frente à exposição diária ao café. Ressaltando a importância do adequado acabamento e polimento para minimizar manchamento do material.

REFERÊNCIAS

1. Anusavice KJ. Phillips - Materiais Dentários, 11.ed. Rio de Janeiro: Elsevier; 2005.

2. Bowen RL. Dental filling material comprising vinyl silane treated fused silica and a binder consisting of the reaction product of bis phenol and glycidyl acrylate U.S. Patent, 3,066,112, 27 Nov, 1962.

3. Bowen RL. Properties of a silica-reinforced polymer for dental restorations. J Am Dent Assoc. 1963;66(1):57-64

4. Polli MJ, Borges $\mathrm{CH}$, Arossi GA. Estabilidade de cor de resina composta frente a corantes da dieta. Rev Iniciação Científica Ulbra. 2014;(12):84-92.

5. Malaspina AO. Avaliação da estabilidade de cor e rugosidade superficial de resinas compostas micro-hibridas, submetidas ao processo de envelhecimento artificial acelerado, em função da fotoativação com lâmpada halógena e LED [tese]. Bauru: Universidade de São Paulo;2009. 
6. Dubinski P, Cardoso AS, Hoeppner MG. Avaliação das causas das substituições de restaurações nas disciplinas de dentística II e clínica integrada do curso de odontologia da UNIPAR-Campus Umuarama. Ci Biol Saúde. 2005;11(1):7-14.

7. Braga SR, Vasconcelos BT, Macedo MR, Martins VR, Sobral MA. Reasons for placement and replacement of direct restorative materialls in Brazil. Quintessence Int. 2007; 38(4):189-94.

8. Pedrini D, Sonoda CK, Poi WR, Barioni SRP, Castro JCM, Brandini DA. Razões Para Substituição de Restaurações em Clínica Integrada. Pesq Bras Odontoped Clin Integr. 2009;9(2):211-15.

9. Fontes ST, Fernández MR, Moura CM, Meireles SS. Color stability of a nanofill composite: effect of different immersion media. J Appl Oral Sci. 2009;17(5):988-91.

10. Lopes LBPM, Araujo ASL, Milagre VB. Quantification of color variation of restorative materials used on pediatric dentistry after pigmentation. RGO Rev Gauch. Odontol. 2015;63(4):383-88.

11. Soares-Geraldo D, Scaramucci T, Steagall-Jr W, Braga SRM, Sobral MAP. Interaction between staining and degradation of a composite resin in contact with colored foods. Braz Oral Res. 2011;25(4):369-75.

12. Carvalho PRB, Filho PFM, Silva CHV. Etiologia e prevenção do manchamento das restaurações estéticas com resinas compostas. IJD. 2003;2(1):236-40.

13.Dauvillier BS, Aarnts MP, Feilzer AJ. Developments in shrinkage control of adhesive restoratives. J Esthet Dent. 2000;12(6):291-99.

14. Brunthaler A, König F, Lucas T, Sperr $W$, Schedle A. Longevity of direct resin composite restorations in posterior teeth. Clin Oral Investig. 2003;7(2):63-70.

15. Manhart J. Direct composite restorations in posterior region: a case history using a nanohybrid composite. Dent Today. 2004;23(11):66,68-70.

16. Gerdolle DA, Mortier E, Droz D. Microleakage and polymerization shrinkage of various polymer restorative materials. $\mathrm{J}$ Dent Child. 2008;75(2):125-33.

17. Boaro LC, Fróes-Salgado NR, Gajewski VE, Bicalho AA, Valdivia AD, Soares $C J$ et al. Correlation between polymerization stress and interfacial integrity of composites restorations assessed by different in vitro tests. Dent Mater. 2014;30(9);984-92.

18. Van Ende A, De Munck J, Lise DP, Van Meerbeek B. Bulk-fill composites: A review of the current literature, J Adhesive Dent. 2017;19(2):95-109.

19. Fronza BM, Rueggeberg FA, Braga RR, Mogilevych B, Soares LES, Martin AA et al. Monomer conversion, microhardness, internal marginal adaptation, and shrinkage stress of bulk-fill resin composites. Dent Mater. 2015;31(12):1542-51

20. Godoi APT. Efeito in situ da associação de chá preto ao uso diário de clorexidina na alteração de cor e rugosidade superficial de resinas compostas [dissertação]. Ribeirão Preto: Universidade de São Paulo; 2009.

21. Samra APB. Avaliação espectrofotométrica da estabilidade de cor de materiais restauradores estéticos [dissertação]. Ponta Grossa: Universidade Estadual de Ponta Grossa; 2004.

22. Yokoyama M. Girotto ML, Ricco RAPO. Estudo comparativo da influência da fotopolimerização por LED e lâmpada halógena no manchamento da resina composta submetida a alimentos corantes. Colloquium Vitae. 2010;2(1):49-53.

23. Caldarelli PG, Beltrani FC, Santos FA, Shibayama R, Hoeppner MG. Manchamento de uma resina composta e sua relação com o efeito da intensidade da luz emitida por aparelhos fotopolimerizadores de lâmpada halógena. Rev Odontol Araçatuba. 2011;32(2):44-48.

24. Shamszadeh S, Sheikh-Al-Eslamian SM, Hasani E, Abrandabadi AN, Panahandeh N. Color Stability of the bulk-fill Composite Resins with Different Thickness in Response to Coffee/Water Immersion. Int J Dent. 2016;2016(4):1-5.

25. Ergucu Z, Turkun LS, Aladag A. Color stability of nanocomposites polished with one-step systems. Oper Dent. 2008;33(4):413-20.

26. Abd Elhamid M, Mosallam R. Effect of bleaching versus repolishing on colour and surface topography of stained resin composite. Australian Dental Journal. 2010;55(4):390-98.

27. Chan KC, Fuller JL, Hormati AA. The ability of foods to stain two composite resins. J Prosthet Dent. 1980;43(5)542-45.

28. Souza E, Espíndola M, Diegoli NM et al. Avaliação da alteração de cor de diferentes compósitos restauradores: Influência de corantes usados na cavidade oral - Estudo in Vitro. RGO. 2006;54(1):43-6.

29. Martini EC, Coppla FM, Reis A et al. Análise da capacidade de remoção de pigmentos da resina composta pelo peróxido de hidrogênio 35\%. Rev Odontol Unesp. 2015;45(1):53-8.

30. Nasser BP, Oliveira TJRS. Avaliação da influência do polimento mediado ou imediato sobre a alteração de cor de uma resina composta micro-híbrida [monografia]. Pindamonhangaba: Faculdade de Pindamonhangaba; 2014.

31. Lopes ES, Linhares TS, Garone Netto N, Lago ADN. Avaliação do efeito de bebidas ao manchamento de resinas compostas. Rev Pesq Saúde. 2016;17(1):147-50.

32. Salgado VE, Rego GF, Schneider LF, Moraes RR, Cavalcante LM. Does translucency influence cure efficiency and color stability of 
resin-based composites? Dent Mater. 2018;34(7):957-66.

33. Manhães GS. Comparação do grau de lisura proporcionado por três diferentes sistemas de acabamento e polimento em resina composta, [monografia]. Londrina: Universidade Estadual de Londrina, Londrina; 2015.

34. Maixner AO, Susin AH. Avaliação da alteração de cor de resina composta submetida à ação de corantes e gêneros alimentícios. Rev Dentística online. 2001;1(2).

35. Oliveira ALBM. Influência do acabamento e polimento na estabilidade de cor e na rugosidade superficial de uma resina composta submetida a diferentes soluções fluoretadas [dissertação]. Araraquara: Universidade Estadual Paulista, Faculdade de Odontologia; 2008.

36. Lu H, Roeder LB, Lei $L$ et al. Effect of surface roughness on stain resistance of dental resin composites. J Est Rest Dent. 2005;17(2):102-8.

37. Oliveira ALBM, Lorenzetti CC, Garcia PPNS et al. Effect of finishing and po1lishing on color stability of a nanofilled resin immersed in different media. Rev Odontol UNESP. 2014;43(5):338-42.

38. Gadonski AP, Feiber M, Almeida L, Naufel FC, Schmitt VL. Avaliação do efeito cromático em resinas compostas nanoparticuladas submetidas a solução café. Rev Odont Unesp. 2018;47(3):137-42.

39. Ertaş $E$, Güler $A U$, Yücel $A C$, Köprülü $H$, Güler E. Color stability of resin composites after immersion in different drinks. Dent Mater J. 2006;25(2):371-76.

40. Topcu FT, Sahinkesen G, Yamanel K, Erdemir U, Oktay EA, Ersahan S. Influence of different drinks on the colour stability of dental resin composites. Eur J Dent. 2009;3(1):50-6.

41. Plaster SC, Peixoto LM, Guedes APA, Silva DN, Castro MCC. Efeito de diferentes fotopolimerizadores e meio de imersão na rugosidade e na cor de um compósito nanoparticulado. Rev Odontol Unesp. 2016; 45(5):283-89.

42. Migot I, Marques AB, Rocha I, Cavalcanti A, Argolo S. Avaliação de cor de diferentes marcas e sistemas de resina composta por espectrofotometria. Rev Bahiana Odonto. 2017; 8(3):68-75.

43. Souza MBA, Oliveira O, Moda MD, Santos PH, Briso ALF, Fagundes TC. Influência de diferentes tipos de fotopolimerizadores e fotoiniciadores na microdureza e cor de resinas compostas. Arch Health Invest 2017; 6(Spec Iss 2):57.

\section{CONFLITO DE INTERESSES}

Os autores declaram não haver conflitos de interesse

\section{AUTOR PARA CORRESPONDÊNCIA}

\author{
Márcia de Almeida Durão \\ Universidade de Pernambuco \\ Faculdade de Odontologia de Pernambuco \\ Av. Gov. Agamenon Magalhães, s/n - Santo Amaro \\ 50100-010Recife-PE, Brasil \\ Email: marciadurao21@gmail.com
}

Submetido em 15/04/2020

Aceito em 23/08/2020 